\title{
Quantitative analysis of pharmaceutical tablets using LIBS-Technique
}

\author{
Naser Almsellati, Omhani Swesi andSabria Aladuli \\ Physics Department, Almargeb University, Libya
}

\begin{abstract}
Laser-induced breakdown spectroscopy (LIBS) is a type of atomic emission spectroscopy which uses a highly energetic laser pulse as the excitation source. In principle, LIBS can analyze any matter regardless of its physical state, solid, liquid or gas. LIBS may be used to evaluate the relative abundance of each constituent element, or to monitor the presence of impurities. The present experiments reveal that in addition to the presence of carbon, hydrogen, nitrogen and oxygen, which can be primarily attributed to the active pharmaceutical ingredients, specific inorganic atoms were also present in all the tablets. Initial attempts at classification by a ratio metric approach using oxygen $(777 \mathrm{~nm})$ to nitrogen $(742.36 \mathrm{~nm}, 744.23 \mathrm{~nm}$ and $746.83 \mathrm{~nm})$ compositional values yielded an optimal value at $746.83 \mathrm{~nm}$ to provide an acceptable classification.
\end{abstract}

Key words: Laser- Spectroscopy- Electron Density- Emission.

\section{INTRODUCTION}

Laser induced breakdown spectroscopy (LIBS) is an analytical method for determination of qualitative and quantitative composition of sample. One of the main advantages of LIBS is the possibility to perform non- contact measurements without significant requirements for sample preparation. LIBS is a versatile method for fast and accurate multi- element analysis of solid, liquid and gaseous samples. In the last few years LIBS has become a popular tool for determination of sample composition and has found versatility in many fields, including environment $^{(\mathbf{1})}$ (Senesi et al., 2009), biomedical ${ }^{(\mathbf{2})}$ (Rehseet al., 2007) and pharmaceutical ${ }^{(\mathbf{3})}$ [Onge et al., 2002].

The pharmaceutical industry is beginning to consider this technique for in-line monitoring and product analysis applications. However, there was not a lot of work published in the literature that deals with the specific application of this technology for pharmaceutical applications $^{(3,4)}$ [Ongeet al., 2002; Ongeet al., 2005].

An important feature of LIBS technique is its speed, which can allow for online deployment. By integrating a peak resulting from the emission of a unique element at a particular wavelength, a signal intensity value proportional to the amount of this particular element present in the material ablated is recorded for each successive laser pulse. This information can be monitored and compared for each site of a tablet for the determination of tablet uniformity distribution, or it can also be compared as an average value between several tablets for content uniformity evaluation.

High energy materials are soft organic compounds with the general formula $\mathrm{C}_{\alpha} \mathrm{H}_{\beta} \mathrm{N}_{\gamma} \mathrm{O}_{v}$. The dominant peaks in LIBS spectra of all such compounds comprise of Carbon, Hydrogen, Oxygen and Nitrogen. They all are exhibit similar molecular and elemental signatures in their LIBS spectra. These molecular species are key signatures of organic substances for identification of organic high energy materials ${ }^{(5,6)}$ (Lazicet al., 2009; Lucenaet al., 2011). 
Naser Almsellati et al.

As sources for material ablation and simultaneous plasma generation from ablated material Q-switched Nd:YAG lasers are commonly used. Intensive laser pulse is focused on the sample surface to produce plasma or laser spark. Emission from the plasma is collected by fiber optic and analyzed by spectrograph or photodiode. When optimal experimental conditions are obtained, LIBS can provide accurate analysis of examined samples.

In this work, we investigate the feasibility of LIBS for routine pharmaceutical tablet investigation such as Parofon 300mg, Parol $500 \mathrm{mg}$ and Aferin $650 \mathrm{mg}$ for compositional information and discrimination among tablets procured over the counter from local pharmacy. Herein, we first report our observations of the LIBS-based elemental analysis on common pharmaceutical tablets. Further, we attempted to classify these tablets into their corresponding functional groups based on a ratio metric approach.

\section{EXPERIMENTAL METHODS}

The over-the-counter drug samples were purchased from a local pharmacy. They were scanned using LIBS with any further processing. The details of the samples are provided in Table (1). For the coated samples, the spectra were first recorded directly with the coating. Here, coated samples refer to the tablets that are available with colored coatings on them.

Table 1: Details of pharmaceutical samples investigated.

\begin{tabular}{|c|c|c|c|}
\hline Sample No. & Sample name & $\begin{array}{c}\text { Formula of the primary } \\
\text { component }\end{array}$ & Ingredients \\
\hline 1 & Parafon $300 \mathrm{mg}$ & $\mathrm{C} 8 \mathrm{H} 9 \mathrm{NO} 2+\mathrm{C} 7 \mathrm{H} 4 \mathrm{ClNO} 2$ & Paracetamol+Chlorzoxazone \\
\hline 2 & Parol $500 \mathrm{mg}$ & $\mathrm{C}_{8} \mathrm{H}_{9} \mathrm{NO}_{2}$ & Paracetamol \\
\hline 3 & Aferine $650 \mathrm{mg}$ & $\mathrm{C}_{8} \mathrm{H}_{9} \mathrm{NO}_{2}+$ & $\begin{array}{c}\text { Paracetamol }+ \\
\text { chlorpheniramine maleate }\end{array}$ \\
\hline
\end{tabular}

Five to ten different pharmaceutical tablets were purchased from a local market. They will be scanned using LIBS with any further processing. LIBS spectra were recorded using a Quantel-Big Sky Nd:YAGlaser (Bozeman, MT, USA), HR 2000 Ocean optics Spectrograph (Dunedin, FL, USA) and Stanford Research System Delay Generator SRS DG535 (Cleveland, $\mathrm{OH}$, USA). The experimental setup Figure (1) shows the LIBS setup. The laser operates at a fundamental wavelength of $532 \mathrm{~nm}$ and used for sample ablation. It was then run in the Qswitched mode at a repetition rate of $1 \mathrm{~Hz}$. External gated detection was performed at $0.5 \mathrm{~ns}$ gate delay and $20 \mathrm{~ns}$ gate width. Spectrograph was externally triggered from the laser at every pulse with the delay generator. Samples were measured by LIBS technique by scanning five different locations and four excitations per location.

A laser-induced breakdown spectroscopy-based analytical methodology is developed to study tablet coating variability in pharmaceutical tablets. The method quantifies the amount of coating on a tablet by assigning an average coating thickness score to it. When tested using samples with different amounts of coating, the coating thickness scores showed direct correlation to the weight gain of the tablet, hence validating the analytical method. The relative significance of the processing parameters and the components of variability were computed using statistical techniques.

The sampling frequency of laser shots was found to have no significant effect. The effect of the position of the laser pulse on the tablet surface was found to be significant; however, it 


\section{Quantitative analysis of pharmaceutical tablets using LIBS-Technique}

was determined that this effect was due to the tablet curvature, which resulted in the laser optical path to intersect the coating diagonally. The variability between batches (lots) manufactured under the same processing conditions was not significant. The largest avoidable source of variability was the tablet-to-tablet component, possibly indicating the inadequate mixing performance of the coating device.

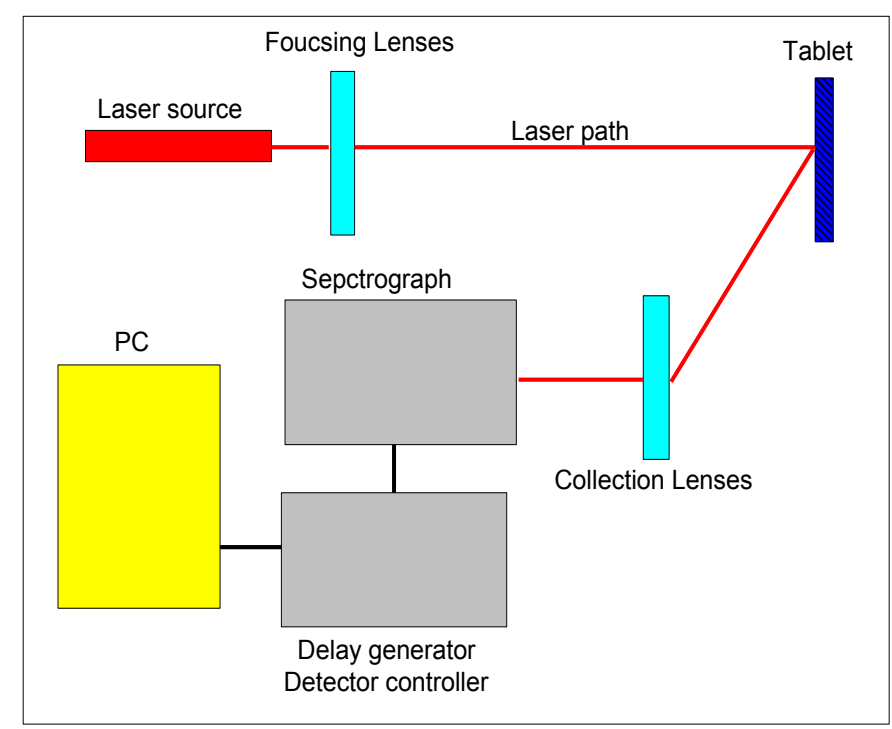

Fig. 1: Experimental set up

\section{Spectral analysis}

\section{RESULTS AND DISCUSSION}

A representative LIBS spectrum for each of the three drug samples was plotted separately in Figure (2), for the shake of clarity. All the spectra exhibited peaks corresponding to nitrogen, oxygen, hydrogen and carbon. These lines can be attributed to the primary components responsible for the action of the tablets (active pharmaceutical ingredients), which are organic molecules. Though the primary component of Aferine does not contain any nitrogen in them, yet their LIBS spectra showed the nitrogen peaks. The possible reason for observing nitrogen peaks in Aferine samples could be the presence of other ingredients such as flavoring and coloring agents that are added to the pharmaceutical tablets. The spectra also exhibited peaks corresponding to, Iron, sodium, magnesium, titanium and calcium. 
Naser Almsellati et al.

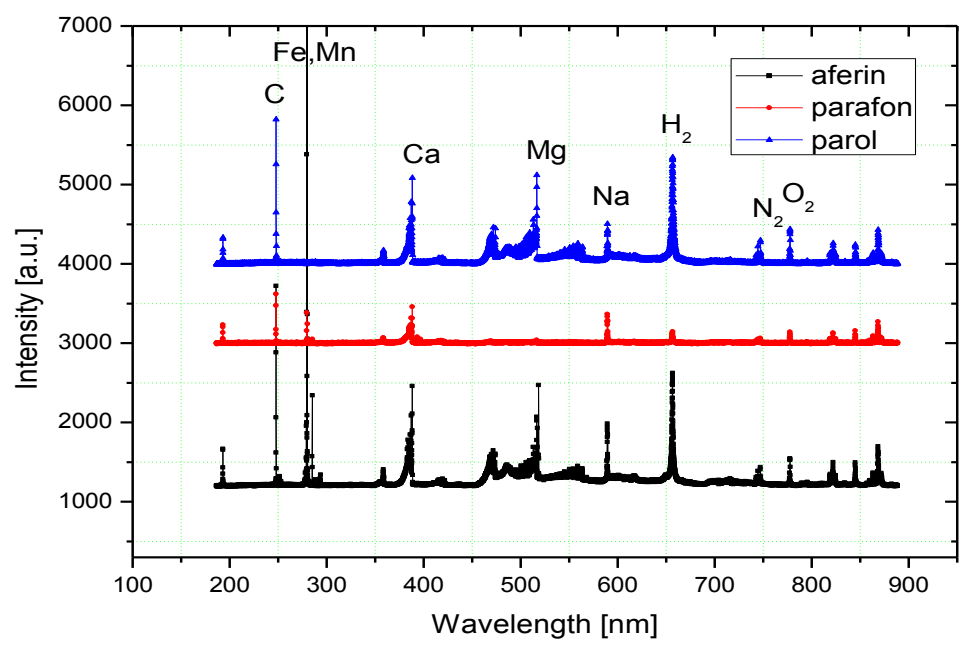

Fig. 2: LIBS spectra of the samples used in the study. (a) Aferin, (b) Parafon, (c) Parol

We suspect that the other metals could be components of excipients (especially sodium) or contaminants. Both the recordings showed peaks corresponding to carbon, hydrogen, oxygen and nitrogen, the coated spectra showed strong peaks corresponding to titanium. Table (2) shows the list of the elements observed in the spectra and their corresponding emission wave lengths.

Table 2: Different peaks observed in LIBS spectra and the corresponding atomic elements

\begin{tabular}{|c|c|c|}
\hline $\begin{array}{c}\text { Sample } \\
\text { No. }\end{array}$ & Element & Wavelength $(\mathbf{n m})$ \\
\hline 1 & Carbon & 247.85 \\
\hline 2 & Iron & $279.78,283.59,285.18$ \\
\hline 3 & Manganese & $279.10,279.48,380.96$ \\
\hline 4 & Sodium & $589.0,589.60$ \\
\hline 5 & Vanadium & $251.16,572.68,635.70$ \\
\hline 6 & Oxygen & $777.19,777.41,777.53,822.18,822.76$ \\
\hline 7 & Nitrogen & $742.36,744.23,746.83,818.48,818.80,821.63,824.23$ \\
\hline 8 & Hydrogen & 656.27 \\
\hline 9 & Magnesium & 518.36 \\
\hline 10 & Titanium & $394.8,395.6,395.8,399.8$ \\
\hline 11 & Calcium & $393.37,396.86$ \\
\hline
\end{tabular}

\section{Ratio metric analysis}

In the present work, the potential of the ratio metric approach was studied for identifying the tablets based on their respective oxygen to nitrogen intensity ratios. This approach was previously shown to yield reasonable identification of organic nitro-compounds, namely4nitroaniline and 4-nitrotoluene by Rai et al. ${ }^{(7)}$. The oxygen peak at $777 \mathrm{~nm}$ (O) (Fig. 3) and nitrogen peaks at $742.36 \mathrm{~nm}$ (N1) and $744.23 \mathrm{~nm}$ (N2) (Figs. $3 \&$ 4) were used for evaluating the 


\section{Quantitative analysis of pharmaceutical tablets using LIBS-Technique}

$\mathrm{O} / \mathrm{N}$ ratios. The oxygen peak at $777 \mathrm{~nm}$ is a triplet and was not fully resolved in our LIBS spectra.

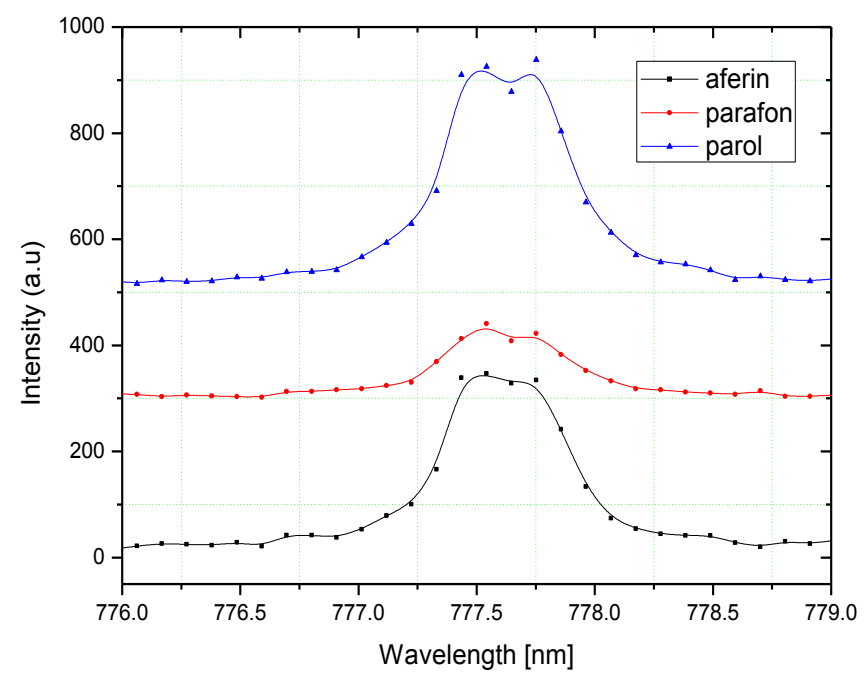

Fig. 3: Oxygen LIBS peaks from (a) Aferin, (b) Parafon, (c) Parol. The dots represent the experimental points

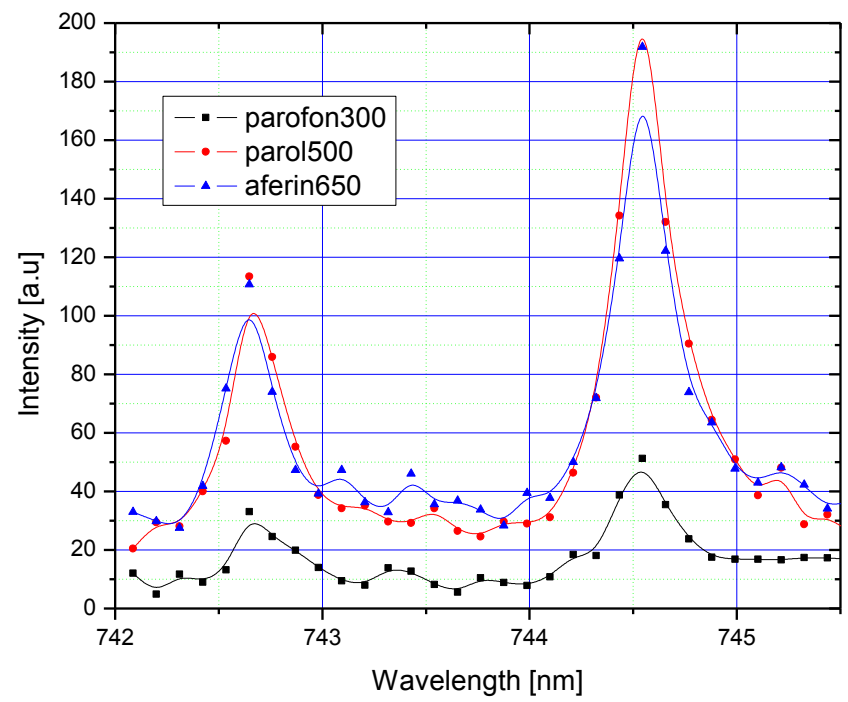

Fig. 4: Nitrogen (N1 and N2) LIBS peaks from (a) Aferin, (b) Parafon, (c) Parol. The dots represent the experimental points

Two different $\mathrm{O} / \mathrm{N}$ ratios were calculated corresponding to the peaks of nitrogen at $\mathrm{O} / \mathrm{N}_{1}$ and $\mathrm{O} / \mathrm{N}_{2}$. A direct evaluation of the $\mathrm{O} / \mathrm{N}$ intensity ratios by considering the areas under the peaks resulted in very large values as shown in Table (3). 
Naser Almsellati et al.

Table 3: Oxygen to nitrogen ratios with the oxygen peak at $777 \mathrm{~nm}(\mathrm{O})$ and nitrogen peaks at $742.36 \mathrm{~nm}(\mathrm{~N} 1)$ and $744.23 \mathrm{~nm}(\mathrm{~N} 2)$.

\begin{tabular}{|c|c|c|c|}
\hline Sample No. & Sample name & $\mathrm{O} / \mathrm{N}_{1}$ & $\mathrm{O} / \mathrm{N}_{2}$ \\
\hline 1 & aferin1_500mg & 4.25 & 2.55 \\
\hline 2 & parofon_500mg & 41.08 & 27.85 \\
\hline 3 & parol_500mg & 23.05 & 12.5 \\
\hline
\end{tabular}

The density of states was calculated for the three tablets for the hydrogen peak at 656.65 $\mathrm{nm}$ (Fig. 5) taking into account the plasma temperature $\mathrm{C}=10000 \mathrm{~K}$. The values of density of states for the three investigated tablets were shown in Figure (6).

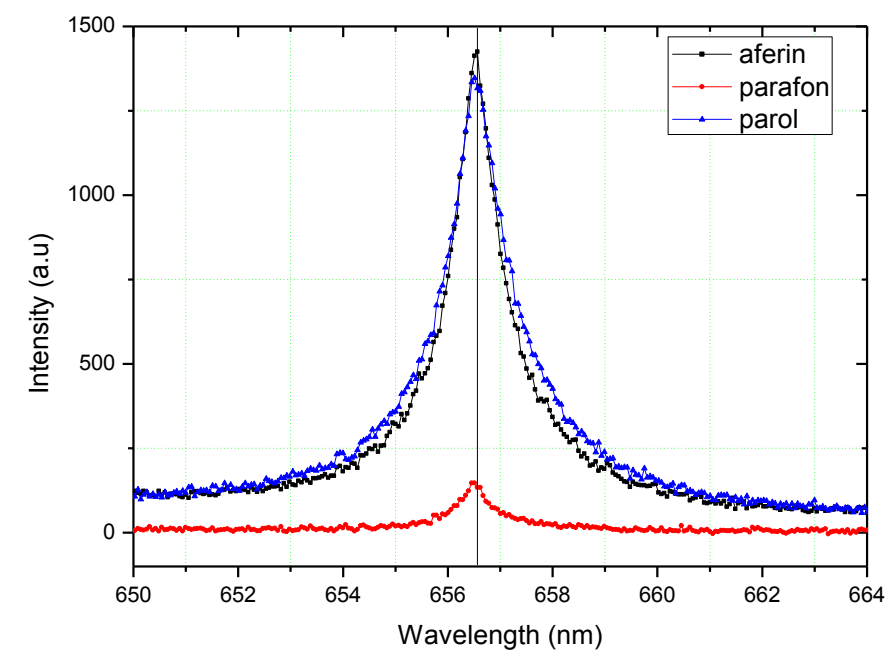

Fig.5. Position of H2peaks for the different tablets 


\section{Quantitative analysis of pharmaceutical tablets using LIBS-Technique}

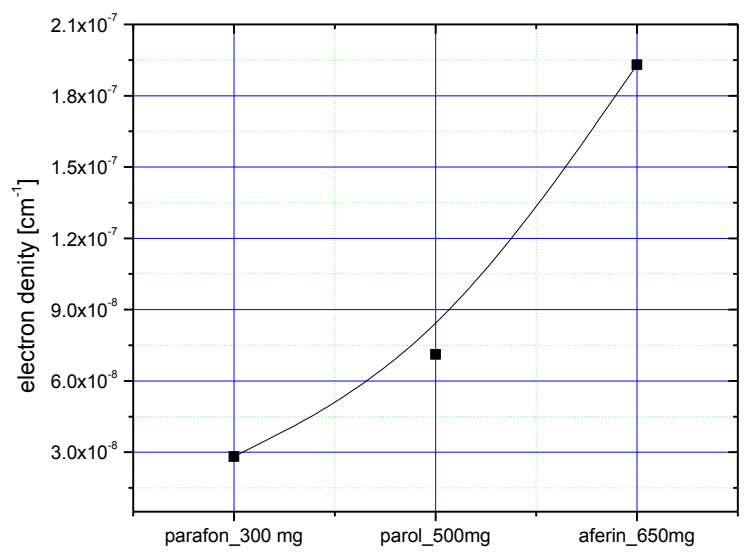

Fig.6: The density of states of the three different tablets (Aferin, Parafon, Parol).

\section{CONCLUSION}

In this work, we have studied the effectiveness of routine monitoring of commercial pharmaceutical tablets using a LIBS technique for routine pharmaceutical tablet investigation such as Parofon $300 \mathrm{mg}$, Parol $500 \mathrm{mg}$ and Aferine $650 \mathrm{mg}$ for compositional information and discrimination among tablets procured over the counter from local pharmacy.

LIBS was evaluated as a rapid and direct method for measuring the drug content in pharmaceutical tablets. Although the tablet components are all organic, in a large fraction of cases the drug molecule contains an element which distinguishes it from the other tablet components. Measuring the drug content can therefore be carried out by quantifying this element.

LIBS can provide new information on the surface and internal distribution of pharmaceutical materials entering into the composition of pharmaceutical solid dosage forms. The technology does not require any sample preparation, samples can be analyzed quickly in less than one minute, and it is specific to the element(s) contained in the chemical structure of drugs and/or other functional materials. Qualitative and quantitative determinations can then be obtained rapidly on drug formulations during the developmental stage or commercial manufacturing.

\section{REFERENCES}

1-Senesi, G. S.; Dell'Aglio, M.; Gaudiuso, R.; De Giacomo, A.; Zaccone, C.; De Pascale, O.; Miano, T.M. and Capitelli, M. (2009). Heavy metal concentrations in soils as determined by laser-induced breakdown spectroscopy (LIBS), with special emphasis on chromium. Environ, Res., 109: 413-420.

2-Rehse, S.J.; Diedrich, J. and Palchaudhuri, S. (2007). Identification and discrimination of Pseudomonas aeruginosa bacteria grown in blood and bile by laser-induced breakdown spectroscopy. Spectrochimica Acta Part B 62 :1169-1176.

3-Onge, St.L.; Kwong, E.; ; Sabsabi, M. and Vadas, E.B. (2002). Quantitative analysis of pharmaceutical products by laser induced breakdown spectroscopy. Spectrochimica, Acta Part B 57: $1131-1140$. 
Naser Almsellati et al.

4-Onge, St.L.; Archambault, J.F.; Kwong, E.; Sabsabi, M. and Vadas, E.B. (2005). Rapid quantitative analysis of magnesium stearate in tablets using laser-induced breakdown spectroscopy. J. Pharm Pharm Sci., 8:272-88.

5-Lazic, V.; Palucci, A.; Jovicevic, S.; Poggi, C. and Buono, E. (2009). Analysis of explosive and other organic residues by laser induced breakdown spectroscopy. Spectrochim, Acta Part B. 64: 1028-1039.

6-Lucena, P.; Doña, A.; Tobaria, L.M. and Laserna, J.J. (2011). New challenges and insights in the detection and spectral identification of organic explosives by laser induced breakdown spectroscopy. Spectrochim, Acta Part B.,66 :12-20.

7- Rai, S.N.; Chopra, R.C., Sharma, K. (2008). Chemical composition and mineral profile of certain sea weeds of Indian coast. Ind. J. Sci., 78(11):1278-1280 